\title{
Pediatric obesity interventions: Are we targeting the right behaviour to impact obesity?
}

\section{Emily Knight}

\author{
University of Western Ontario
}

One quarter of Canadian children are overweight or obese, ${ }^{1}$ a situation that poses serious consequences for Canada's health care system, both now and in the future. The World Health Organization estimates that 500,000 deaths in North America and Western Europe result from obesity and related diseases, and considers suboptimal weight status (i.e., overweight and obesity) to be among the greatest health challenges and risk factors for disease in the twenty-first century. ${ }^{2}$ In Canada, the economic cost of obesity and physical inactivity is estimated to be $\$ 9.6$ billion annually, which is nearly $5 \%$ of Canadian health care costs. ${ }^{3}$ Physical inactivity is a preventable risk factor for many obesity-related chronic and non-communicable diseases, including diabetes. ${ }^{4,5}$ Therefore, promoting healthy physical activity (PA) behaviours, particularly early in life, represents a substantial opportunity for chronic disease prevention, health care cost savings, and improved quality of life. ${ }^{2}$

Regular participation in moderate and vigorous intensity PA is associated with important physical, cognitive and emotional benefits, including musculoskeletal development, maintenance of healthy body weights, prevention of high blood pressure, and social and mental development. ${ }^{6}$ Therefore, healthy PA behaviours have implications for children's psychological, sociological, and physiological health and development. ${ }^{7}$ According to Canadian physical activity guidelines, ${ }^{8}$, children and youth should accumulate at least 60 minutes of moderate to vigorous PA daily to accrue health benefits. However, directly measured data (via accelerometer) on a nationally representative sample of Canadian children indicate that merely $9 \%$ of boys and $4 \%$ of girls are meeting this target. ${ }^{9}$ Furthermore, it has been demonstrated that physical fitness behaviours track from childhood into adulthood, ${ }^{7}$ suggesting that the fitness of today's children is likely a reflection of future fitness behaviours, and corresponding health status, of the Canadian adult population. The bad news is that today's children are less fit than children were just a generation ago, ${ }^{1}$ which has significant implications for public health in the years to come. To improve the health of future generations, the current obesity and PA crisis should be addressed. Based on the current state of the literature, the purpose of this commentary is to discuss how PA has been employed as a strategy in pediatric obesity interventions and to present ideas for better targeting PA behaviours in future interventions to reduce obesity.

PA is defined as any activity that involves bodily movement and results in energy expenditure. ${ }^{10}$ Therefore the PA spectrum encompasses a range of activities children can engage in on a daily basis (Figure 1) - inactivity, such as sleeping; sedentary activity, such as reading or playing computer games; light activity, such as playing with toys; and moderate and vigorous intensity activities, such as playing tag in the schoolyard, skateboarding or swimming. When measuring children's engagement in daily PA behaviours it is important to consider the range of activities that children can participate in and the associated health benefits or consequences of these activities. Yet interventions for obese children and adolescents typically only attempt to increase and measure the time spent engaged in moderate and vigorous PA, which is only one end of the daily PA spectrum. This narrow focus may be contributing to the limited success that has been realized through typical obesity interventions designed for children and adolescents., ${ }^{5,11,12,13}$ Obesity and PA interventions to date have been plagued by high dropout rates ${ }^{11}$ and have been shown to be ineffective, resulting in no change or even undesirable changes in PA participation and/or body composition. ${ }^{13}$ Interventions to date have perhaps missed an opportunity to observe changes in PA behaviours and obesity status by limiting their PA measurement to only moderate and vigorous PA.

Indeed, it has been suggested in the literature that targeting a 


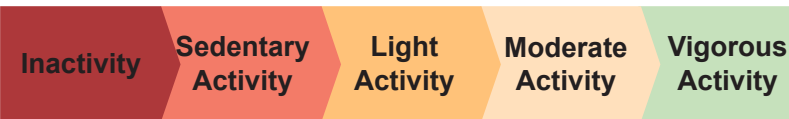

Figure 1: The continuum of daily physical activities ranges from inactivity through sedentary, light, moderate and vigorous intensity PA. On a daily basis children might engage in sports, games or active play that fall anywhere along the PA spectrum.

reduction in time spent engaged in lower intensity activities, instead of increasing time spent engaged in moderate and vigorous PA, may be a more effective approach to changing both PA behaviours as well as obesity status.,14 Given that the average Canadian child spends $62 \%$ of their waking hours engaged in sedentary behaviours and less than $8 \%$ of their day participating in high-intensity $\mathrm{PA}^{9}{ }^{9}$ reducing time spent engaged in sedentary pursuits represents a substantial opportunity for changing PA behaviour through obesity interventions. For example, substituting sedentary activities in children's daily routines (such as being driven to school or sitting for extensive periods of time at a computer) with light intensity activities (such as walking to school or standing periodically while using the computer) may be a more effective way to change PA behaviours and impact obesity. It may not be reasonable to expect that a child or adolescent who currently spends the majority of their daily routine engaged in sedentary pursuits will immediately run or swim or engage in similar high-intensity activities. Therefore, interventions may achieve greater success in reducing obesity and increasing PA behaviours if they are designed to target and measure the range of PA intensities children engage in on a daily basis.

In summary, literature available from pediatric obesity interventions employing a PA component indicates that these interventions typically target and measure changes in total moderate and vigorous PA. However, future research should consider the entire spectrum of PA behaviours that children can engage in on a daily basis when designing and implementing PA-based pediatric obesity interventions. Findings from such studies may provide insight for future health promotion strategies to prevent or change obesity and develop healthy PA behaviours among Canadian children.

\section{References}

1. Tremblay M, Shields M, Laviolette M, Craig C, Janssen I, Connor Gorber S. Fitness of Canadian children and youth: Results from the 2007-2009 Canadian health measures survey. Health Reports. 2010;21[1]:1-14.

2. WHO. The World Health Report 2002: Reducing risks, promoting healthy life. Geneva: World Health Organization 2002.

3. Katzmarzyk $P$, Janssen I. The economic costs associated with physical inactivity and obesity in Canada: An update. Canadian Journal of Applied Physiology. 2004;29[1]:90-115.

4. Owen N, Sparling P, Healy G, Dunstan D, Matthews C. Sedentary behavior: emerging evidence for a new health risk. Mayo Clinic Proceedings. 2010;85(12):1138-41.

5. Lau DCW, Douketis JD, Morrison KM, Hramiak IM, Sharma AM, Ur E. 2006 Canadian clinical practice guidelines on the management and prevention of obesity in adults and children [summary]. Canadian Medical Association Journal. 2007;176(8):S1-S13.

6. Janssen I, LeBlanc AG. Systematic review of the health benefits of physical activity and fitness in school-aged children and youth. International Journal of Behavioral Nutrition and Physical Activity. 2010;7[1]:40.

7. Hills A, King N, Armstrong T. The Contribution of Physical Activity and sedentary behaviours to the growth and development of children and adolescents: implications for overweight and obesity. Sports Medicine. 2007;37(6):533-45.

8. CSEP. Canadian physical activity guidelines. Ottawa: Canadian Soceity for Exercise Physiology 2011.

9. Colley R, Garriguet D, Janssen I, Craig C, Clarke J, Tremblay M. Physical activity of Canadian children and youth: Accelerometer results from the 2007 to 2009 Canadian Health Measures Survey. Health Reports. 2011;22[1]:1-10.

10. Caspersen C, Powell K, Christenson G. Physical activity, exercise, and physical fitness: Definitions and distinctions for health-related research. Public Health Reports. 1985;100[2]:126-31.

11. Reinehr T, Widhalm K, l'Allemand D, Wiegand S, Wabitsh M, Holl R. Twoyear follow-up in 21,784 overweight children and adolescents with lifestyle intervention. Obesity. 2009;17:1196-9.

12. Kamath C, Vickers K, Ehrlich A, McGovern L, Johnson J, V S, et al. Behavioral interventions to prevent childhood obesity: A systematic review and meta-analyses of randomized trials. Journal of Clinical Endocrinology \& Metabolism. 2008;93:4606-15.

13. Hagstromer M, Elmberg K, Marild S, Sjostrom M. Participation in organized weekly physical exercise in obese adolescents reduced daily physical activity. Acta Paediatrica. 2009;98:352-254.

14. Epstein L, Roemnich J, Paluch R, Raynor H. Physical Activity as a substitute for sedentary behavior in youth. Annals of Behavioural Medicine. 2005;29(3):200-9.

\section{Emily Knight}

Emily Knight is a MPT/PhD student in Health \& Rehabilitation Sciences at University of Western Ontario. Her research interests are in healthy active living across the life span, and her doctoral research focuses on the use of technology and persuasive design in health behaviour prescription. 